\title{
Differences between Italian and immigrant female citizens' hospitalizations related to obstetrical and gynecological diseases in the province of Ferrara, Italy
}

\author{
C Alessandro, B Mauro
}

\section{Citation}

C Alessandro, B Mauro. Differences between Italian and immigrant female citizens' hospitalizations related to obstetrical and gynecological diseases in the province of Ferrara, Italy. The Internet Journal of Gynecology and Obstetrics. 2009 Volume 12 Number 2.

\begin{abstract}
Introduction - In the recent years, the province of Ferrara, in the Italian Northeast has been characterized by the phenomenon of massive immigration. The aim was to study hospital admissions related to the immigrant female population and its implications. Materials and Methods - The Local Healthcare Unit of the National Health Service provided data related to hospital admissions between 01/01/2006 to 31/12/2006. Results and Conclusions - On 53699 admissions of female subjects, 2656 were related to immigrants. A total of 1509 admissions were obstetrical or gynecological hospitalizations. The high number of DRGs representative of spontaneous abortions and voluntary interruptions of pregnancy may be an expression of social problem of some sections of the immigrant people community. The dissemination of information on the existence of social assistance programs is important. This can prevent the occurrence of critical health situations, especially during pregnancy and childbirth.
\end{abstract}

\section{BACKGROUND AND AIM OF THE WORK}

The territory of the province of Ferrara, in north-eastern Italy has been in recent years characterized by the phenomenon of mass migration of people from countries especially outside the European Union [1]. This led to differing health needs compared to the Italian population one. This could be a result from the differences in demographic characteristics of the two populations, such as the lower mean age of the immigrant population. The present work is aimed to identify areas of possible interventions allowing optimization of resources in the light of possible changes over time.

\section{MATERIALS AND METHODS}

The Local Healthcare Unit (LHU) of Ferrara provided data related to the hospital admissions from 01/01/2006 to $31 / 12 / 2006$ granted to immigrant and Italian citizens. The database included nationality, date of birth, residence. The gender was inferred from the tax code. From a health-related point of view, the database showed data related to the type of access, the type of admission (ordinary or day hospital (DH)), the days of hospitalization, the hospital facility, the DRG (Version 19). The facilities included those directly controlled by LHU, the University Hospital, private structures, and extra-provincial structures. GynecologicalDRG included those from 353 to 369 included and obstetrical-DRG included those from 370 to 384 . Access 2003 [2] and Excel 2003 [3] were used to manage and analyse data.

\section{RESULTS}

There were 91370 admissions (ordinary and DH). The number of admissions for Italian patients were 87581 (males 36538 (41.7\%), females 51043 (58.3\%)); and for immigrants were 3789 (males 1133 (29.9\%), females 2656 (70.1\%)). With regard to the type of housing, there were slight differences $(\mathrm{p}<0.001)$ among Italians (ordinary 71.5\%, DH $28.5 \%$ ) and immigrants (ordinary 72.5, DH 27.5). With regard to females, a total of 53699 hospitalizations was observed: 51043 were related to Italian patients $(95.1 \%)$ while 2656 (4.9\%) to immigrant patients. Out of a total of 10345 hospitalizations for obstetric or gynecological DRG performed in $2006,8836(85.4 \%)$ were granted to Italian patients and $1509(14.6 \%)$ to immigrant patients $(\mathrm{p}<0.001)$. The ratio between ordinary and $\mathrm{DH}$ hospitalization (ordinary/DH) was 0.601 in Italian patients and it was 0.497 in immigrant patients $(\mathrm{p}<0.001)$. The distribution by age of hospitalized patients is shown in Table 1. 


\section{Figure 1}

Table 1 - Distribution by age classes of gynecological or obstetrical hospitalizations in 2006 in province of Ferrara: female population

\begin{tabular}{|c|c|c|c|c|c|c|c|c|c|c|c|c|c|c|c|c|}
\hline \multicolumn{17}{|c|}{ Age clases } \\
\hline & 0.9 & $10-14$ & 15.40 & 20.24 & 2520 & 30.34 & 35.39 & 4044. & $45-40$ & 50.54 & 55.50 & 60.84 & 6560 & $70-74$ & 75- & \\
\hline & 0 & 18 & 138 & 433 & 1138 & 2040 & $18 \mathrm{~A} 7$ & 875 & 549 & 457 & 328 & 254 & 294 & 197 & 262 & \\
\hline ime & 0 & 3 & 79 & 289 & 425 & 345 & 193 & 34 & 43 & 29 & 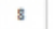 & 1 & 9 & 7 & 0 & 180 \\
\hline & 0 & 21 & 213 & 722 & 1563 & 2391 & 2042 & 939 & 592 & 496 & 336 & 255 & 299 & 204 & 262 & \\
\hline
\end{tabular}

Table 2 shows the comparison amongst the major obstetrical and gynecological DRG, sorted by absolute frequencies, in Italian and immigrant population.

\section{Figure 2}

Table 2 - Hospitalizations divided by DRGs

\begin{tabular}{|r|l|c|c|c|}
\hline DRGs & & Italian & Immigrant & Total \\
\hline 373 & Vaginal Delivery w/o Complic Diagnoses & 1548 & 372 & 1920 \\
381 & Abortion wo D\&C & 1004 & 367 & 1371 \\
359 & Uter, Adnex Pr, Ca In Situ/Non-Malig w/o CC & 1254 & 58 & 1312 \\
364 & D\&C, Conization Except for Malignancy & 1188 & 56 & 1244 \\
371 & Cesarean Section w/o CC & 689 & 134 & 823 \\
360 & Vagina, Cervix \& Vulva Procedures & 711 & 62 & 773 \\
383 & Oth Antepartum Dx w Med Complications & 360 & 129 & 489 \\
384 & Oth Antepartum Dx w/o Med Complications & 389 & 36 & 425 \\
379 & Threatened Abortion & 247 & 68 & 315 \\
369 & Menstrual, Other Female Repro Syst Disor & 260 & 48 & 308 \\
380 & Abortion wo D\&C & 145 & 42 & 187 \\
374 & Vaginal Delivery W Steril \& Or D\&C & 138 & 26 & 164 \\
382 & False Labor & 116 & 18 & 134 \\
370 & Cesarean Section w CC & 89 & 20 & 109 \\
356 & Female Repro System Reconstructive Proc & 83 & 2 & 85 \\
372 & Vaginal Delivery W Complic Diagnoses & 59 & 13 & 72 \\
378 & Ectopic Pregnancy & 52 & 19 & 71 \\
366 & Malignancy, Female Repro System w CC & 67 & 3 & 70 \\
355 & Uter, Adnex Proc Non-Ov/Adnex Malig w/o CC & 65 & 2 & 67 \\
358 & Uter, Adnex Pr, Ca In Situ Non-Malig w CC & 52 & 4 & 56 \\
357 & Uter, Adnex Proc for Ovar, Adnexal Malig & 48 & 3 & 51 \\
361 & Laparoscopy, Incisional Tubal Interrupt & 47 & 4 & 51 \\
363 & D\&C, Conization, Radio-Implant, for Malig & 45 & 1 & 46 \\
368 & Infections, Female Repro System & 33 & 9 & 42 \\
365 & Other Female Repro System O.R. Proc & 35 & 1 & 36 \\
353 & Pelvic Evise, Rad Hysterect \& Rad Vulvect & 33 & 2 & 35 \\
367 & Malignancy, Female Repro System w/o CC & 32 & 3 & 35 \\
376 & Postpart \& Post Abort Dx w/ O.R. Proc & 18 & 4 & 22 \\
377 & Postpart \& Post Abort Dx w O.R. Proc & 17 & 2 & 19 \\
354 & Uter, Adnex Proc Non-Ov/Adnex Malig w CC & 9 & 0 & 9 \\
362 & Endoscopic Tubal Interruption & 3 & 0 & 3 \\
375 & Vagin Deliv W O.R Proc Ex Steril \&Or D\&C & 0 & 1 & 1 \\
Total & & 8836 & 1509 & 10345 \\
\hline
\end{tabular}

Table 3 shows the admissions divided by facility.

\section{Figure 3}

Table 3 - Hospitalizations divided by facility

\begin{tabular}{|c|c|c|c|c|c|}
\hline & LHU & Outside province & Universitay Hospital & Private & Total \\
\hline Italian & 3649 & 1123 & 3858 & 206 & 8836 \\
Immigrants & 659 & 91 & 756 & 3 & 1509 \\
Total & 4308 & 1214 & 4614 & 209 & 10345 \\
\hline
\end{tabular}

\section{DISCUSSION}

Ferrara is aligning to many other Italian cities in the goal to assess the real health needs of the immigrant population. Few years ago, the admissions made by immigrants were less than a $1 \%$ [4]; at present they overcame the $4 \%$ threshold. Considering both sexes, we have more accesses from females in percentage. This seems to be in contradiction with the classical theory that predict that an early immigration is composed by young adult males [5]. This is explainable by the fact that in Italy the practice of employment of women to help elderly people in daily needs is widespread. Most of these people are migrant women from Eastern Europe. The lower median age of immigrant patients compared to Italian ones (32.5 years vs 55.8 years) is explainable in presence of a recent migratory phenomenon, associated with a low number of familiar reunions. Regarding the causes of hospitalizations, we focused on the large number of DRGs 380 and 381 which represents spontaneous abortions and mainly voluntary interruptions of pregnancy. This large amount of induced abortions may be the expression of a social problem in the immigrant population related to the difficulties in interrupting work in order to complete the pregnancy. Another possible explanation could be the lack of resources to care for and to raise children. There is a need to improve the immigrant population's awareness of social care programs and perspectives (often ignored), in terms of guarantee of the rights to work during and after the pregnancy. The dissemination of information of such programs should be early so that it can be activated in the early months of pregnancy to ensure a bettert assistance. This could avoid both the induced abortion and the occurrence of critical adverse health situations during pregnancy and childbirth. The high incidence of ectopic pregnancies and complications of peripartum period amongst the immigrants is noteworthy. With regard to cancer, the immigrant population, probably because of the lower average age, has a lower incidence than the Italian population. The use of healthcare facilities seemed geared to a greater use of the facilities located in the province compared to the Italian population. We also found lack of utilisation of private facilities: this may highlight a significant confidence in public healthcare facilities, or lower utilisation of the higher cost private facilities by the usually lower income immigrants.

\section{CONCLUSIONS}

The present data show a new perspective of state of health in Ferrara. The differences between the health resource utilisation by the Italian population and the growing immigrant population require a comprehensive assessment. There should be greater awareness of the existence of social programs amongst the immigrants. This can prevent the occurrence of critical health situations, especially during pregnancy and childbirth and improve care for the 
immigrants.

\section{References}

1. www.fieri.it International and European immigration research forum. The immigration in Italy. [accessed on October 29, 2007]

2. Access 2003. Redmond, U.S.A.: Microsoft, 2001
3. Excel 2003. Redmond, U.S.A.: Microsoft, 2001

4. Laroche M. Health status and health services utilization of Canada's immigrant and non-immigrant populations.

Canadian Public Policy 2000; 26(2), 51-75

5. Mosca F. Osservatorio sull'immigrazione della Provincia di Ferrara - Rapporto 2006 [Immigration in the province of Ferrara - 2006 report]. Ferrara: Province of Ferrara, 2007 


\section{Author Information}

Cucchi Alessandro, (MD, PhD)

Department of Clinical and Experimental Medicine, Section of Hygiene and Occupational Health, University of Ferrara

Bergamini Mauro, (MD, Prof)

Department of Clinical and Experimental Medicine, Section of Hygiene and Occupational Health, University of Ferrara 